\title{
Parametric sensitivity analysis of a mathematical model of facultative mutualism
}

\author{
Enu Ekaka-a, ${ }^{1}$ E.C. Nwachukwu, N.M. Nafo, I.A. Agwu, \\ Department of Mathematics and Computer Science, Rivers State University of Science and Technology, Port \\ Harcourt, Rivers State, Nigeria \\ Department of Mathematics and Statistics, University of Port Harcourt, Nigeria \\ Department of Mathematics and Computer Science, Rivers State University of Science and Technology, Port \\ Harcourt, Nigeria \\ Department of Mathematics, Abia State Polytechnic, Aba, Abia State, Nigeria
}

Abstract: The complex dynamics of facultative mutualism is best described by a system of continuous non-linear first order ordinary differential equations. The methods of 1-norm, 2-norm, and infinity-norm will be used to quantify and differentiate the different forms of the sensitivity of model parameters. These contributions will be presented and discussed.

Accepted Date: 06 June 2013

\section{Introduction}

According to Morin (2002), competition and predation have been observed as two dominant types of interaction between species. In the literatures of mathematical ecology or mathematical biology, competition is the most recognised type of interaction while mutualism despite its relevance seems to be not frequently mentioned in most research agenda. Having conducted a detailed literature review on the topic of the dynamics of transitions between population interactions, using a non-linear $\alpha$-function, we found that the application of parametric sensitivity analysis of the model parameters for this important model is rarely introduced as a method of studying the importance of the model parameters of such model. We are motivated to implement the tool of parametric sensitivity analysis because it is the first stage in the process of developing a deterministic model which describes the dynamics of two interacting populations. To the best of our knowledge, we are yet to see any reported mathematical analysis which involves the utilization of a numerical parametric sensitivity analysis. This method is expected to estimate and classify the sensitivity of model parameters which were proposed by Hernandez (1998). In the next section, we will present the mathematical formulation of the model on which our analysis id based.

\section{Mathematical Formulation}

Following Hernandez (1998), we consider the following complex system of two continuous nonlinear first order ordinary differential equations of the following mathematical structure:

$$
\begin{aligned}
& \frac{d N_{1}}{d t}=r_{1} N_{1}\left[1-\frac{N_{1}}{K_{1}}+\left(\frac{b_{1} N_{2}-c_{1} N_{2}^{2}}{1+d_{1} N_{2}^{2}}\right) \frac{N_{2}}{K_{1}}\right] \\
& \frac{d N_{2}}{d t}=r_{2} N_{2}\left[1-\frac{N_{2}}{K_{2}}+\left(\frac{b_{2} N_{1}-c_{2} N_{1}^{2}}{1+d_{2} N_{1}^{2}}\right) \frac{N_{1}}{K_{2}}\right]
\end{aligned}
$$

Here, $N_{1}$ and $N_{2}$ are populations of specie 1 and specie 2 respectively, $r_{1}$ and $r_{2}$ are growth rates of specie 1 and specie 2 respectively, $K_{1}$ and $K_{2}$ are carrying capacities of specie 1 and specie 2 respectively. The parameters $b_{1}, b_{2}, c_{1}, c_{2}, d_{1}$ and $d_{2}$ modify the general shape and represent the environmentalinfluence on $\alpha_{i j}$ that is, the "exogenous effect".

This facultative model (Hernandez (1998)) is driven by thirteen (13) parameters. To conduct a realistic and systematic parametric sensitivity analysis of this model, we have proposed to study the sensitivity analysis of the two intrinsic growth rates $\left(r_{l}\right.$ and $\left.r_{2}\right)$, carrying capacities $\left(K_{l}\right.$ and $\left.K_{2}\right)$ and the initial conditions $\left(N_{l}(0)\right.$ and $\left.N_{2}(0)\right)$ for obvious ecological or biological reasons. In the next section, we will describe the key relevant materials and methods which will enable us to solve this problem numerically. 


\section{Materials and Methods}

Following Ford et al (2010) and Hernandez (1998), these researchers in mathematical biology have identified similar types of interactions between species such as mutualism, commensalism, parasitism or predation and competition. The method of Ford et al (2010) was based on using the biological ideas to construct a model for two competing plant species in a harsh climate whereas the model of Hernandez (1998) simply describes in great detail the undergoing transitions between different types of partially positive interaction hereby called facultative and obligate mutualism which is consistent with the classification of Morin $(2002,187-189)$.

In our recent literature review, the current state of the spread of knowledge clearly shows that the work of Hernandez (1998) has been popularly cited by 32 other researchers. However, none of these researchers has attempted to study the parametric sensitivity analysis of the facultative model.Hernandez (1998) considered an interaction where both $\alpha$-functions are of the $\alpha(+,-)$ type and reported that at low densities, the association is mutualistic but at higher densities the same association was considered to be competitive. It was also reported in the same paper that depending on the threshold value where $\alpha_{12}$ and $\alpha_{21}$ are equal to zero, immediate density values can be experimentally determined for a situation where a parasitic interaction can also occur. Part of developing this model involves assuming that both species can exist either alone or in association. The approximate model formulation was constructed on the basis of these simplifying assumptions. The method of sensitivity analysis which we have used in this study has been adapted from recent research reports of Ekaka-a (2009), Ekaka-a et al (2012) and Nwachukwu and Ekaka-a (2013). A brief sketch of this method is as follows:

STEP I: Code the given system of continuous non-linear first order ordinary differential equation in a Matlab programing language.

STEP II: Modify and code a similar program which is used for a variation of a single parameter one-at-a-time while other model parameters are fixed.

STEP III: Design an appropriate ODE45 Runge-Kutta scheme which will simulate the program in step I and step II

STEP IV: Use the program in step III to calculate the 1-norm, 2-norm, and infinity norm of three solution trajectories in the same manner, use the same program to calculate the three popular norms of the differences of the solution trajectories.

STEP V: Based on the original parameter in the step I, calculate the cumulative percentage effect on the solution trajectories due to variation of each chosen parameter at a time when other parameters are fixed.

STEP VI: Interprete the result quantitatively. That is, the parameter which when varied a little and produces the biggest cumulative effect on the solution trajectories is called a most sensitive.

\section{Results andDiscussion}

In this section, we will present and discuss our results which we have achieved in this study. In this section, the notation CV stands for the popular statistical coefficient of variation.

Table 1 Percentage variations of $\mathrm{r}_{1}$

\begin{tabular}{|c|c|c|c|c|c|}
\hline $\begin{array}{c}\text { variations } \\
\text { mn }\end{array}$ & $\mathbf{0 . 0 1 5}$ & $\mathbf{0 . 0 3}$ & $\mathbf{0 . 0 4 5}$ & $\mathbf{0 . 0 6}$ & $\mathbf{0 . 0 7 5}$ \\
\hline 1-norm & 10.5213 & 5.3085 & 3.5391 & 2.6518 & 2.1221 \\
\hline 2-norm & 10.9065 & 7.5868 & 6.0936 & 5.1905 & 4.5654 \\
\hline$\infty$-norm & 32.5020 & 30.7169 & 29.1662 & 27.6902 & 26.5351 \\
\hline
\end{tabular}

Table 2 Percentage variations of $\mathrm{r}_{2}$

\begin{tabular}{|c|c|c|c|c|c|}
\hline $\begin{array}{c}\text { variations } \\
\text { mn }\end{array}$ & $\mathbf{0 . 0 1 5}$ & $\mathbf{0 . 0 3 0}$ & $\mathbf{0 . 0 4 5}$ & $\mathbf{0 . 0 6}$ & $\mathbf{0 . 0 7 5}$ \\
\hline 1-norm & 18.5029 & 9.1881 & 6.0497 & 4.4794 & 3.5403 \\
\hline 2-norm & 22.8126 & 15.5895 & 12.3174 & 10.3293 & 8.9562 \\
\hline$\infty$-norm & 98.7772 & 88.7117 & 81.2441 & 74.9255 & 69.5011 \\
\hline
\end{tabular}

Table 3 Percentage variations of $\mathrm{K}_{1}$

\begin{tabular}{|c|c|c|c|c|c|}
\hline $\begin{array}{c}\text { variations } \\
\text { mn }\end{array}$ & $\mathbf{0 . 0 1}$ & $\mathbf{0 . 0 2}$ & $\mathbf{0 . 0 3}$ & $\mathbf{0 . 0 4}$ & $\mathbf{0 . 0 5}$ \\
\hline 1-norm & 65.2987 & 64.9995 & 64.6973 & 64.3887 & 64.0780 \\
\hline 2-norm & 47.5824 & 47.3460 & 47.1080 & 46.8654 & 46.6221 \\
\hline$\infty$-norm & 54.2792 & 54.0124 & 53.7196 & 53.4540 & 53.1737 \\
\hline
\end{tabular}


Table 4 Percentage variations of $\mathrm{K}_{2}$

\begin{tabular}{|c|c|c|c|c|c|}
\hline $\begin{array}{c}\text { variations } \\
\text { mn }\end{array}$ & $\mathbf{0 . 0 1}$ & $\mathbf{0 . 0 2}$ & $\mathbf{0 . 0 3}$ & $\mathbf{0 . 0 4}$ & $\mathbf{0 . 0 5}$ \\
\hline 1-norm & 65.4713 & 65.1745 & 64.8735 & 64.5666 & 64.2571 \\
\hline 2-norm & 47.5803 & 47.3472 & 47.1114 & 46.8715 & 46.6304 \\
\hline$\infty$-norm & 62.0988 & 61.9169 & 61.6954 & 61.4953 & 61.2576 \\
\hline
\end{tabular}

Table 5 Percentage variations of $\mathrm{IC}_{1}$

\begin{tabular}{|c|c|c|c|c|c|}
\hline $\begin{array}{c}\text { variations } \\
\text { mn }\end{array}$ & $\mathbf{0 . 0 4}$ & $\mathbf{0 . 0 8}$ & $\mathbf{0 . 1 2}$ & $\mathbf{0 . 1 6}$ & $\mathbf{0 . 2 0}$ \\
\hline 1-norm & 0.5121 & 0.5079 & 0.5028 & 0.5022 & 0.5013 \\
\hline 2-norm & 5.5154 & 5.4601 & 5.4051 & 5.3500 & 5.2949 \\
\hline$\infty$-norm & 74.1298 & 73.5982 & 73.0003 & 72.3749 & 71.7193 \\
\hline
\end{tabular}

Table 6 Percentage variations of $\mathrm{IC}_{2}$

\begin{tabular}{|c|c|c|c|c|c|}
\hline $\begin{array}{c}\text { variations } \\
\text { mn }\end{array}$ & $\mathbf{0 . 1}$ & $\mathbf{0 . 2}$ & $\mathbf{0 . 3}$ & $\mathbf{0 . 4}$ & $\mathbf{0 . 5}$ \\
\hline 1-norm & 1.3768 & 1.3709 & 1.3681 & 1.3499 & 1.3443 \\
\hline 2-norm & 13.8051 & 13.6737 & 13.5407 & 13.4063 & 13.2710 \\
\hline$\infty$-norm & 117.6685 & 116.9752 & 116.1566 & 115.2819 & 114.3127 \\
\hline
\end{tabular}

Table 7 results of coefficient of variation for model parameters

\begin{tabular}{|c|c|}
\hline parameter $\mathbf{r}_{\mathbf{1}}$ & $\mathbf{C V}$ \\
\hline 1-norm & 0.9640 \\
\hline 2-norm & 0.4310 \\
\hline --norm & 0.0838 \\
\hline
\end{tabular}

\begin{tabular}{|c|c|}
\hline parameter $\mathbf{K}_{\mathbf{1}}$ & $\mathbf{C V}$ \\
\hline 1-norm & 0.0075 \\
\hline 2-norm & 0.0081 \\
\hline$\infty$-norm & 0.0082 \\
\hline
\end{tabular}

\begin{tabular}{|c|c|}
\hline parameter IC $_{\mathbf{1}}$ & $\mathbf{C V}$ \\
\hline 1-norm & 0.0091 \\
\hline 2-norm & 0.0162 \\
\hline$\infty$-norm & 0.0132 \\
\hline
\end{tabular}

\begin{tabular}{|c|c|}
\hline parameter $\mathbf{r}_{\mathbf{2}}$ & $\mathbf{C V}$ \\
\hline 1-norm & 1.0038 \\
\hline 2-norm & 0.4678 \\
\hline o-norm & 0.1481 \\
\hline
\end{tabular}

\begin{tabular}{|c|c|}
\hline parameter $\mathbf{K}_{\mathbf{2}}$ & $\mathbf{C V}$ \\
\hline 1-norm & 0.0074 \\
\hline 2-norm & 0.0080 \\
\hline o-norm & 0.0054 \\
\hline
\end{tabular}

\begin{tabular}{|c|c|}
\hline parameter IC $_{\mathbf{2}}$ & $\mathbf{C V}$ \\
\hline 1-norm & 0.0104 \\
\hline 2-norm & 0.0157 \\
\hline o-norm & 0.0115 \\
\hline
\end{tabular}

V. Discussion of results

From our results which have been presented in the previous section, we observe that the two carrying capacities can be classified as the most sensitive parameters using the 1-norm and 2-norm estimated sensitivity values while the two initial conditions can be classified as relatively least sensitive parameters. However, the $\infty$ norm estimated value shows that the second initial condition is clearly a more sensitive parameter than any of the other five model parameters. In the context of measuring the best estimate of sensitivity, we can clearly see that the infinity norm is the best estimate for measuring the sensitivity of model parameters such as $r_{1}, r_{2}$, and $K_{2}$ while the 1norm is the best estimate for measuring the sensitivity of model parameters such as $K_{1}$ and the initial conditions.

\section{Conclusion}

In terms of further parameter estimation to minimize uncertainty in model prediction, carrying capacities and the growth rates should be targeted for the purpose of model re-validation. In this study, the initial conditions in the main should be considered as rough estimates. In our further research, we propose to study the sensitivity of other seven (7) parameters which we have not considered in this present study.

\section{References}

[1]. Ekaka-a E. N. (2009): Computational and Mathematical Modelling of Plant Species interactions in a Harsh Climate. PhD Thesis, Dept. of Mathematics, The university of Liverpool and The University of Chester, United Kingdom.

[2]. Ford Neville J, Lumb Patricia M, Ekaka-a Enu (2010): Mathematical modelling of plant species interactions in a harsh climate, Journal of Computational and Applied Mathematics, Vol. 234, pp. 2732-2744.

[3]. Hernandez MJ (1998): Dynamics of transitions between population interactions: a nonlinear interaction alpha-function defined, Proceedings of the Royal Society B Biological Sciences London 1998 265, 1433-1440.. 
[4]. Nwachukwu E. C. and E. N. Ekaka-a (2013): Sensitivity Analysis using a partially coupled system of differential equations without delay, Journal of Mathematics and System Science (ISSN 2159-5291, USA).

[5]. Morin P.J (2002), Community Ecology, Blackwell Publishing Company, United Kingdom. 